\title{
The Relation between Structure and Properties of Crystalline Polymers
}

\author{
Leo MANDELKERN \\ Department of Chemistry and Institutes of Moelcular Biophysics, \\ Florida State University, Tallahassee, Florida 32306, U.S.A.
}

(Received August 20, 1984)

\begin{abstract}
The influence of both structure and crystallization conditions on a variety of properties characteristic of semi-crystalline polymers are discussed. A careful distinction needs to be made between molecular structure and the independent structural variables which describe the crystalline state. This set of independent variables includes the degree of crystallinity; the structure of the non-crystalline regions; the crystallite thickness distribution; the structure and relative amount of interface; the crystallite structure and the supermolecular structure. The dependence of these variables on molecular structure and crystallization conditions are examined in detail as is their influence on properties. By following this procedure the influence of molecular structure on properties is deduced. A variety of thermodynamic, spectral and mechanical properties are analyzed by these methods.
\end{abstract}

KEY WORDS Structural Variables / Degree of Crystallinity / Crystallite Thickness / Supermolecular Structure / Interfacial Structure / Adjacent Recently / Mechanical Properties / Raman Spectroscopy /

Studies of the crystallization behavior of polymers have proceeded sufficiently far so as to enable an analysis to be made of the influence of structure on properties. Two distinctly different types of structure needs to be carefully distinguished. On the one hand there is the concern with the influence of molecular or chain structure. In this case the principle variables are the chain length, for molecular weight fractions, the polydispersity, for whole polymers and the structural regularity of the chain. In addition the structural variables which define the crystalline state also have to be given consideration. We shall be concerned here with how these independent structural variables are influenced by the important elements of molecular structure and by the crystallization conditions. The question then arises as to how these structural variables influence properties. These include thermodynamic, spectroscopic and mechanical properties as well as other macroscopic ones. By following this procedure the influence of molecular structure will be established. Thus, although we shall be considering both kinds of structure they will be carefully delineated.
A set of independent structural variables have been identified which either individually or in particular combinations contribute to and control a specific property. The variables which have been thus identified are: the level, or degree, of crystallinity; the structure of the residual non-crystalline region; the crystallite thickness distribution; the structure and relative amount of the interfacial region; the crystallite structure; and the supermolecular structure. We shall examine each of these structural variables. Emphasis will be given to their meaning, method of determination how they are influenced by molecular structure and crystallization conditions. Then their influence on properties will be considered. The experimental examples to be discussed have been selected predominantly from among the polyethylenes. However, studies with other polymers are sufficiently far advanced to substantiate the generality of the results and conclusions.

\section{RESULTS AND DISCUSSION}

It is instructive at the outset to consider the 
influence of molecular weight, when fraction are used, on the crystallization process itself. We do so by examining the isothermal crystallization from the melt of a wide molecular weight range of linear polyethylene fractions. ${ }^{1,2}$ These results, which are important to the task at hand, are summarized in Figure 1. Here the extent of the transformations, or the degree of crystallinity, $(1-\lambda(t))$ is plotted against the log time for crystallization at $126.1^{\circ} \mathrm{C}^{* 1}$. The isotherm for each molecular weight has been shifted along the horizontal axis to give the superposed set of curves that are illustrated. The solid line (devoid of experimental points) represents the theoretical Avrami isotherm for a substance which is completely transformed. All of the fractions, irrespective of molecular weight, begin to crystallize in exactly the same way and, initially quantitatively adhere to the Avrami formulation. ${ }^{1,2}$ It is clear, however, that the transformation of polymers is far from being complete. The extent of the transformation, i.e. the degree of crystallinity that can be attained at the isothermal crystallization temperature is very dependent on the molecular weight. In this example it ranges from about 0.80 at the lower molecular weights to 0.35 for $M=1.2 \times 10^{6}$. The level of crystallinity can be reduced even more when the molecular weight is increased further. ${ }^{2}$ Thus, there is a very definite and important influence of molecular weight on the level of crystallinity that can be attained.

A more detailed discussion of the factors which influence the level of crystallinity will be given in the next section. For the present we wish to focus attention on the important fact that the crystallization process is effectively completed after the major portion of the transformation has occurred. The log time scale of Figure 1 reflects the fact that only an imperceptible amount of crystallinity develops in the "flat" region of the isotherm in the real time domain. The crystallinity level at which the process essentially ceases is dependent on the molecular weight. Irrespective of theories of crystallite growth that might be offered, whether involving segmental motion, reptation, or other processes the experimental fact remains that crystallization is halted. Since the transformation for all molecular

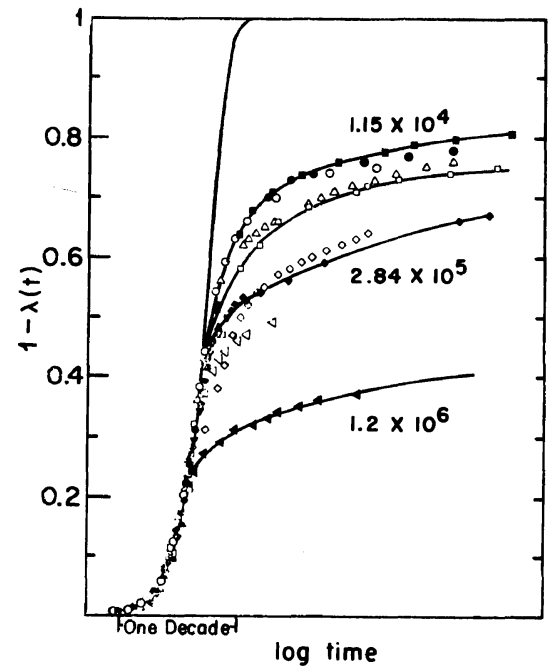

Figure 1. Plot of degree of crystallinity, $1-\lambda(t)$, as a function of log time (on arbitrary scale) for molecular weight fractions of linear polyethylene. Key molecular weights indicated in plot. Crystallization temperature $126.1^{\circ} \mathrm{C}$. From ref 1 .

weights starts in exactly the same manner the kinetics, as illustrated in Figure 1, shows that the structure, or topology, of the residual melt acts to retard the crystallization process. Although difficult to explicitly define at present these structural factors are very dependent or the initial molecular weight prior to the onset of crystallization. They, therefore, must be given very specific attention when considering the molecular influence on properties. Thus, there are at least two main effects of molecular weight: (a) the level of crystallinity that can be attained and (b) the structure of the residual non-crystalline regions.

\section{Degree of Crystallinity $1-\lambda$}

The concept of the degree of crystallinity was introduced very early in the study of crystalline polymers. However, the discovery of lamellar-like crystallites lead to the once widely held view that the concept of a degree of crystallinity was incorrect. This idea was placed in disrepute and banished to obscurity. ${ }^{4-7}$ It was postulated that liquid-like and crystalline regions did not co-exist. The well known deviation in thermodynamic and spectral properties

*1 Similar results have been obtained over all isothermal crystallization temperature for linear polyethylene ${ }^{1,2}$ and for polyethylene oxide. ${ }^{3}$ 
from that of the unit cell were attributed to internal defects and contributions from the crystallite interface. More detailed studies of properties, ${ }^{8-10}$ analysis of small angle neutron scattering patterns ${ }^{11-14}$ as well as thin section electron microscopy ${ }^{15-19}$ of bulk crystallized polymers has made it abundantly clear that the lamellar crystallites are connected to each other by chain units which have a disordered liquid-like structure. It is somewhat ironical that modern electron microscopy clearly demonstrates the existence of a substantial noncrystalline region located exterior to the crystallite core. ${ }^{17-19}$ The concept of the degree of crystallinity has been experimentally demonstrated and quantitatively re-established for a number of different polymers.

The quantitative nature of the degree of crystallinity concept can be better understood by examining the influence of molecular constitution and crystallization conditions. The major differences in $1-\lambda$ that can be achieved by varying the molecular weight at the crystallization temperature were illustrated in Figure 1. Similar results have been found with polyethylene oxide, ${ }^{20}$ trans-polyisoprene, ${ }^{20,21}$ and poly(tetramethyl phenylene siloxane) (TMPS) ${ }^{20,22}$ Upon cooling to ambient temperature, from the crystallization temperature, further crystallization occurs. Although on a relative basis more crystallization occurs at the higher molecular weights a profound influence of chain length still remains. ${ }^{1,23}$ In addition to the polymers just cited the degree of crystallinity has been quantitatively described for polyolefins, polyesters, polyamides and cis-polyisoprene (natural rubber) to cite a few more examples. The level of crystallinity of natural rubber is well established to be about $0.30 . .^{24,25}$ This value is consistent with the results for other polymers because of the very high molecular weight involved. The quantitative data currently available indicates that the degree of crystallinity approaches a limiting value of $0.2-0.3$ with high molecular weight. ${ }^{20,26}$

The introduction of structural, or chemical, irregularities into the chain causes major changes in the generalizations deduced for homopolymers. In contrast to the crucial influence of the molecular weight on the degree of crystallinity of homo-

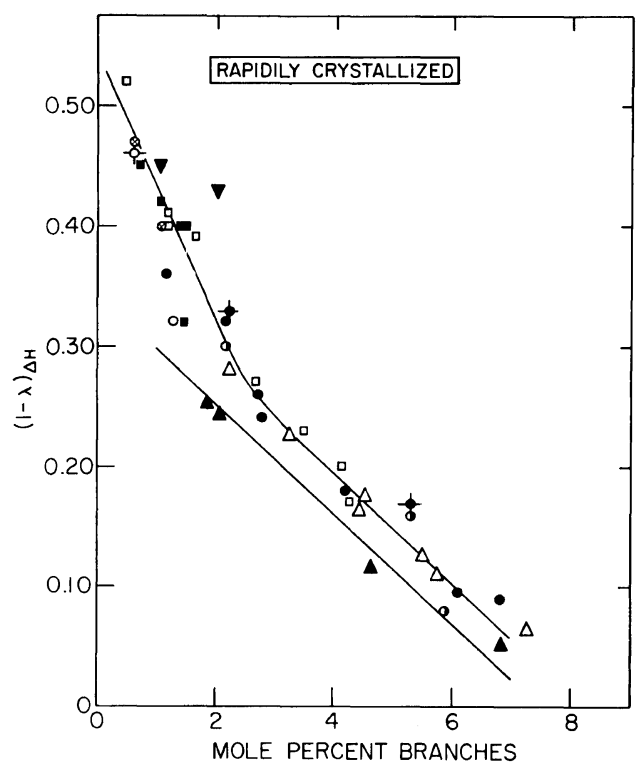

Figure 2. Plot of degree of crystallinity from enthalpy of fusion, $(1-\lambda)_{\Delta H}$ for ethylene copolymers. Hydrogenated polybutadiene $\triangle$; ethylene-butene $\square$; diazoalkane, $n$-propyl $\boldsymbol{\Delta}$; ethylene-octene $\boldsymbol{\square}$; ethylenepropylene $\boldsymbol{\nabla}$; ethylene-vinyl acetate $\mathbf{0}, \mathbf{0}$; branched polyethylene $\bigcirc, \bigcirc, \otimes$. Data from ref $33,56,83$, and 85 .

polymers, chain length has almost no effect in copolymers except in the extremes of very low and very high molecular weights. ${ }^{2}$ Examples of the influence of the co-unit type on the degree of crystallinity are summarized in Figure 2 for a set of rapidly crystallized ethylene copolymers which possesses a close to random sequence distribution. These data have been compiled from a variety of sources and here $1-\lambda$ has been determined by enthalpy of fusion measurements. Except for those samples which contain directly bonded methyl groups and the $n$-propyl branched copolymers, prepared by the copolymerization of the appropriate diazoalkanes ${ }^{27}$ all of the chains behave in a similar manner. Setting aside the exceptions for the moment, it is found that the initial introduction of structural irregularities causes a rapid decrease in $1-\lambda$. This decreases continues with added co-unit content until the crystallinity, at ambient temperature, eventually disappears. Except for the special cases noted it is significant

\footnotetext{
*2 For the purposes of analyzing the crystallization behavior of polymers we must recognize that any structural or chemical irregularity causes the chain to behave as a copolymer. ${ }^{27}$
} 
that the results are independent of the chemical nature of the side-group. This result is expected from theory if the co-unit is effectively excluded from the crystal lattice. ${ }^{28,29}$ Thus, side groups as diverse as ethyl and acetate behave in exactly the same way.

It has been well established from melting temperature-composition studies that for the ethylene-propylene type copolymers the methyl side group enters the lattice on an equilibrium basis. ${ }^{27,30}$ Therefore, for these copolymers somewhat higher levels of $1-\lambda$ are to be expected, as is in fact observed. The molecular weights of homopolymers and copolymers prepared by the decomposition of the diazoalkanes are the order of several million. ${ }^{23,27}$ In analogy with the homopolymer results for this extreme in molecular weight we anticipate a somewhat lower level of crystallinity, for corresponding co-unit contents.

Other methods for determining the level of crystallinity, as for example the density, or analysis of the Raman internal modes, ${ }^{31,32}$ show a very similar pattern of results. ${ }^{33} \mathrm{We}$, therefore, find that in the polyethylenes, and presumably in other polymers as well, it is possible to obtain values for the level of crystallinity which range from about 0.90 , or slightly greater at one extreme to just a trace at the other.

Although the degree of crystallinity is demonstrated to be a quantitative concept and the different methods display the same functional behavior, a direct comparison shows that there are small but significant differences between techniques. For example in Figure $3 a$ and $3 b$ a comparison is made between the degree of crystallinities obtained from density and enthalpy of fusion. These data represent the widest range values that can be attained. The compilation for the linear polymers is presented in Figure 3a, while the data for the branched polymers and copolymers is given in Figure 3b. Except for the very high levels of crystallinity, where good agreement is obtained, $(1-\lambda)_{d}$ is always found to be slightly greater than $(1-\lambda)_{\Delta H}$. These small differences can be attributed to the contribution of the interfacial enthalpy because of the thin crystallites usually formed. ${ }^{34}$ The density measurements, on the other hand, include the interfacial contribution. This conclusion is substantiated by analysis of the Raman internal modes. ${ }^{31,32}$ In this method the degree of crystallinity, $\alpha_{c}$, is

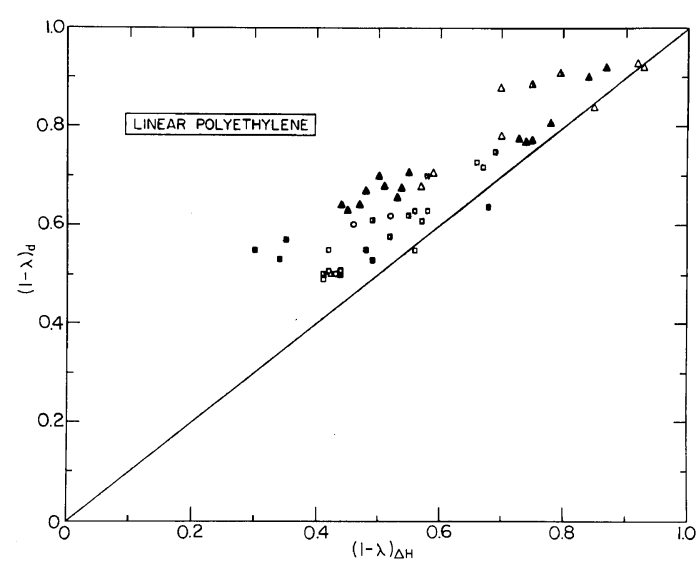

(a)

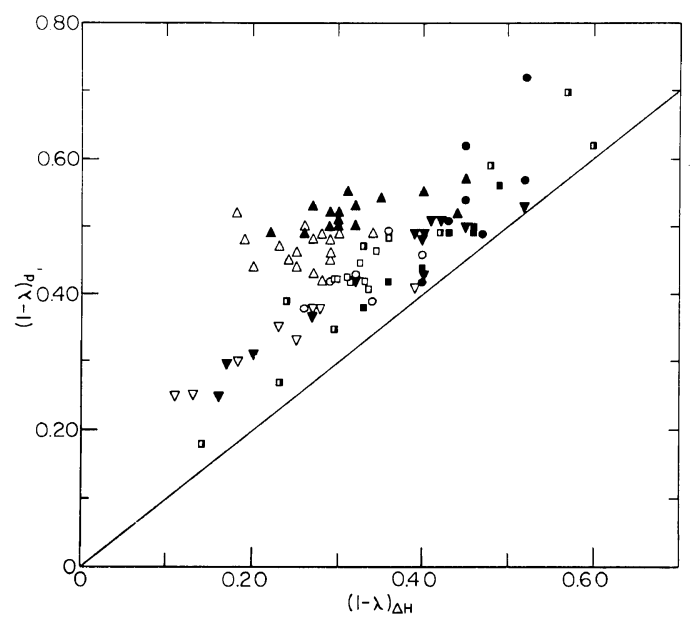

(b)

Figure 3. Plot of degree of crystallinity obtained from density $(1-\lambda)_{d}$ against value obtained from the enthalpy of fusion; $(1-\lambda)_{\Delta H}$. (a) Linear polyethylene; from ref $56 \triangle, \square$; from ref $85 \Delta, \square$; from ref $83 \bigcirc$, from 80 $\Delta, \square$. (b) Copolymers and branched polyethylene. From ref $80 \triangle, \boldsymbol{\Delta}$; from ref $79 \square$; from ref $56 \square$; from ref $83 \bigcirc$; from ref $85 \bigcirc$; from ref $67 \nabla$; from ref $33 \boldsymbol{\nabla}$; from ref 98 a.

obtained from the integrated intensity of the $A_{\mathrm{g}}$ component of the $\mathrm{CH}_{2}$ bending mode at $1416 \mathrm{~cm}^{-1}$. This procedure yields the relative amount of the orthorhombic crystal and is unencumbered by any contribution from the interface. A comparison of $\alpha_{c}$ with $(1-\lambda)_{\Delta H}$ is given in Figure 4 for a large number of different type polyethylene samples. We note that $\alpha_{c}$ and $(1-\lambda)_{\Delta H}$ are identical for the linear polyethylenes over the accessible range of about 0.4 


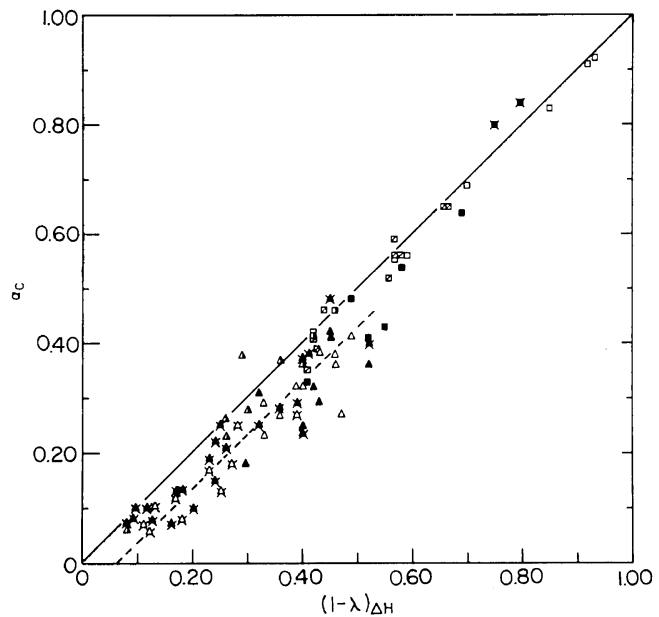

Figure 4. Plot of degree of crystallinity $\alpha_{c}$, as determined from Raman inrernal modes against $(1-\lambda)_{\Delta H}$ for linear and branched polyethylene and ethylene copolymers. From ref $56 \square, \square, \triangle$; from ref 85 . $\mathbf{a}, \mathbf{\square}, \mathbf{\Delta}$; from ref 83; from ref $67 \varnothing$; from ref $33>$

to 0.9 . However, for the copolymers and branched polymers the magnitude of $(1-\lambda)_{\Delta H}$ is always about $5 \%$ greater than $\alpha_{c}$. This small difference results from the fact that structurally irregular chains have a very broad melting range. Hence, $(1-\lambda)_{\Delta H}$ includes a measure of the small amount of crystallinity which has already disappeared at room temperature while $\alpha_{c}$ does not include this contribution. The magnitude of the crystallinity involved is only about $5-10 \%$. From the results of Figure 4 we can conclude that the main reason for the difference between $(1-\lambda)_{d}$ and $(1-\lambda)_{\Delta H}$ is the inclusion of the interfacial contribution to the measured density.

As has been mentioned previously thin section electron microscopy makes clear that disordered liquid-like regions are associated with the lamellar crystallites. ${ }^{1-19}$ In fact a quantitative analysis of such electron micrographs for linear polyethylene ${ }^{19}$ as well as ethylene copolymers ${ }^{35}$ shows that the values of $1-\lambda$ that are obtained are in good agreement with those from density and enthalpy of fusion measurements.

\section{Interfacial Region}

Since lamellar-like crystallites are universally observed in homopolymer crystallization attention has been focused on the interfacial structure that is associated with the basal planes. At one time it was widely believe that these crystallites were comprised of regularly folded chains which formed a very welldefined smooth interface. These conclusions were based solely on direct microscopic observations. ${ }^{4,6,7,36}$ Subsequently, the kinetic theory of chain folding was developed in an attempt to give these deductions a theoretical basis. ${ }^{37-39}$ The substance of the argument was that nuclei comprised of regularly folded chains developed into mature crystallites while the initial molecular structure was maintained. This concept was based solely on the principles of classical nucleation theory. It has been pointed out quite clearly by Price, ${ }^{40}$ and subsequently by others, ${ }^{10,41,42}$ that the concept that critical sized nuclei are composed of regularly folded chain is an assumption that is inserted into the theory. It is very definitely not a deduction of theory. The formalism that is developed will hold for any type chain structure within the nucleus. General nucleation theory, and the temperature coefficient derived from it, are unfortunately not unique to a particular nucleus structure. ${ }^{10,43}$

A recent report has clarified earlier work which interpreted the infrared spectra of crystallites comprised of mixed hydrogenated and deuterated linear polyethylene. ${ }^{44,45}$ It has now been concluded from these measurements that a folded chain structure is not present in bulk crystallized polyethylene. This conclusion is in accord with wide angle neutron scattering studies for similarly constituted mixed systems. ${ }^{46}$

It should be recognized, in retrospect, that there is no substantive experimental evidence, or theoretical basis, for assuming a regularly folded chain crystallite. On the other hand there is no problem in satisfying the observations of lamellar crystallites and a nucleation controlled crystallization process, with other interfacial structures. The observation of lamellae does not require regularly folded chains. The lamellar crystallite habit results from the spatial requirement of the polymer chains. The sequence of ordered chain units cannot be dissipated abruptly at the basal plane for most of the crystal structures found in polymers. ${ }^{47}$ The flux of chains emanating from the crystal surface cannot become isotropic at the sharp boundary. Consequently, there must be an interfacial region, or zone, wherein the crystalline order is dissipated. The analysis of properties $^{8-10}$ and of small angle neutron scattering pat- 
terns ${ }^{12,13}$ requires the presence of an irregularly structured interfacial zone. Major problems that are being currently addressed are concerned with the properties of the chain units which traverse crystallites, as well as the sequence distribution of those chains that reenter the crystallite from which they emanate. These of course are not problems in nucleation theory and are not resolved by its application. In a major theoretical achievement ${ }^{48,49}$ it has recently been shown for polyethylene that within the equilibrium interphase which exists between the ordered crystalline region and the isotropy of the liquid state about $70 \%$ of the chains reenter the same lamellar from which they emerge. Of these, less than about $20 \%$ are in an adjacent reentry position. Most of the remainder occur in nearby sites. The thickness of the interface is calculated to be about $12 \AA$ which is in good accord with experiment. ${ }^{50-52}$ These conclusions are for the situation where the ordered sequences are aligned normal to the basal plane and equilibrium applies. However, when the chain axis is inclined to the basal plane, as is the usual situation, the extent of adjacent reentry will be further reduced. For real crystalline systems, which represent a non-equilibrium situation, the extent of adjacent reentry will be still less. An increase in the interfacial layer can be anticipated.

The magnitude of the interfacial region in the polyethylenes can be obtained from broad line proton NMR,,$^{53,54}$ and from an analysis of the Raman internal modes. ${ }^{55,57}$ A summary of the results obtained by the latter method are given in Table I. For molecular weights equal to, or less than, about $(1.5-2) \times 10^{5}$ the interfacial content is very modest, being about $5 \%$, or less. For the higher molecular weights, irrespective of the crystallization mode, the interfacial content is of the order of about $10 \%$ and has become more significant.

For chains containing structural irregularities which are excluded from the lattice the number of sequences that can participate in the crystallization are limited. Consequently, in addition to the reduced level of crystallinity a more extensive interfacial region, richer in co-unit content is to be expected. The data presented in Figure 5 support this expectation. The introduction of only a small concentration of structural irregularities is sufficient to develop an appreciable interfacial content, $\alpha_{\mathrm{b}}$. It rapidly approaches the order of $20 \%$ and is an appreciable portion of the system. Except for the
Table I. Interfacial content, $\alpha_{b}$, for linear polyethylenes ${ }^{\mathbf{a}, \mathbf{b}}$

\begin{tabular}{|c|c|c|c|}
\hline $\begin{array}{l}\text { Polymer } \\
\text { sample }\end{array}$ & $M_{w} \times 10^{-3}$ & $\begin{array}{l}\text { Crystallization } \\
\text { conditions }\end{array}$ & $\alpha_{\mathrm{b}}$ \\
\hline 1 & 1.14 & Quenched, $-78^{\circ} \mathrm{C}$ & $0 \pm 4$ \\
\hline 2 & 2.05 & Quenched, $-78^{\circ} \mathrm{C}$ & $-2 \pm 5$ \\
\hline \multirow[t]{2}{*}{3} & 27.8 & Quenched, $100^{\circ} \mathrm{C}$ & $5 \pm 4$ \\
\hline & & Quenched, $-129^{\circ} \mathrm{C}$ & $6 \pm 5$ \\
\hline 4 & 188.5 & Quenched, $-129^{\circ} \mathrm{C}$ & $5 \pm 5$ \\
\hline \multirow[t]{2}{*}{5} & 316.0 & Slow cooled & $10 \pm 5$ \\
\hline & & Quenched, $-129^{\circ} \mathrm{C}$ & $12 \pm 6$ \\
\hline 6 & 428.0 & Quenched, $-129^{\circ} \mathrm{C}$ & $11 \pm 6$ \\
\hline \multirow[t]{2}{*}{7} & $1,620.0$ & Quenched, $-78^{\circ} \mathrm{C}$ & $10 \pm 6$ \\
\hline & & $\begin{array}{l}\text { Isothermal } \\
\left(130^{\circ} \mathrm{C} \text { for } 4 \text { weeks }\right)\end{array}$ & $11 \pm 5$ \\
\hline \multirow[t]{2}{*}{8} & 180.0 & Quenched, $95^{\circ} \mathrm{C}$ & $8 \pm 5$ \\
\hline & & Quenched, $-129^{\circ} \mathrm{C}$ & $7 \pm 6$ \\
\hline \multirow[t]{2}{*}{9} & $8,000.0$ & Slow cooled & $13 \pm 5$ \\
\hline & & Quenched, $-129^{\circ} \mathrm{C}$ & $11 \pm 6$ \\
\hline
\end{tabular}

a Data from ref 56.

b Samples 1-7 molecular weight fractions; samples 8 and 9 unfractionated.

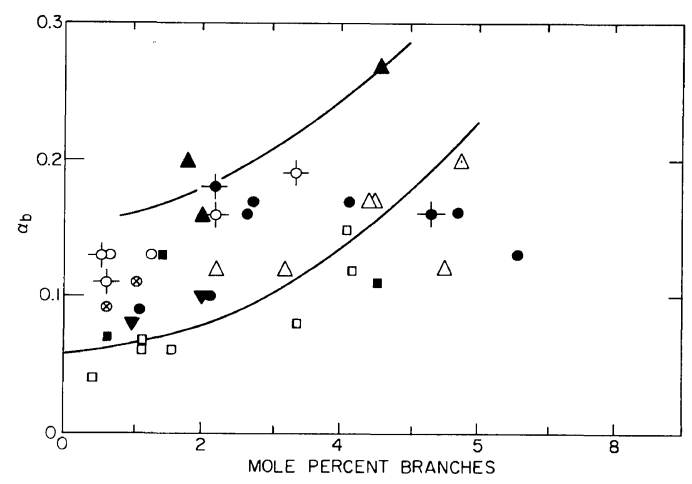

Figure 5. Plot of fraction interface, $\alpha_{b}$, against mole percent branches for ethylene copolymers. From ref 33 $\triangle, \square, \boldsymbol{\Delta}, \boldsymbol{\mathbf { Q }}, \boldsymbol{\bigcirc}$; from ref $83 \bigcirc$; from ref $85 \otimes, \boldsymbol{\nabla}$, from ref $56-\dot{-}-$

very high molecular weight diazoalkane copolymers, the specific chemical nature of the co-unit does not play a major role. Thus, very high molecular weights are no longer required to develop an appreciable interfacial content. The very high molecular weight copolymer that are represented in this figure have a higher interfacial content than the corresponding low molecular weight samples.

Based on the previous discussion of the level of crystallinity we would expect that the sum $\alpha_{c}+\alpha_{b}$ 


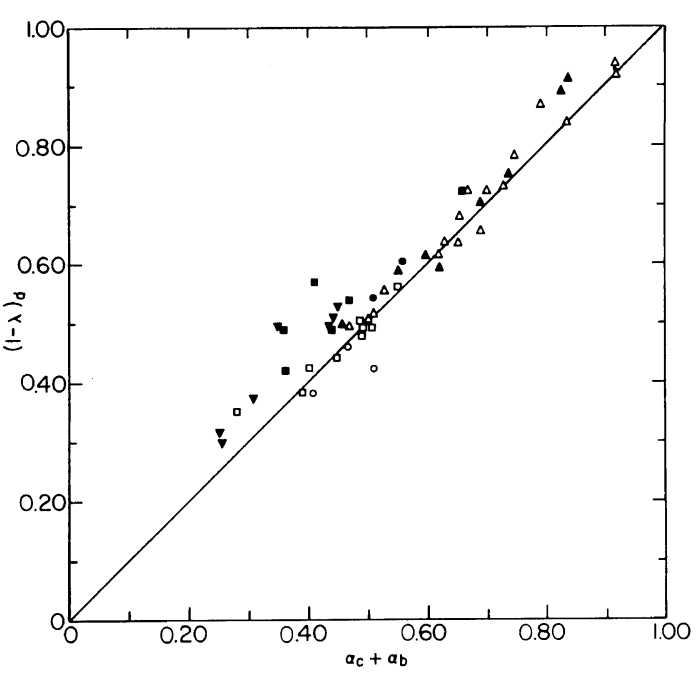

Figure 6. Plot of $(1-\lambda)_{d}$ against some of $\alpha_{c}+\alpha_{b}$ for linear and branched polyethylenes and ethylene copolymers. From ref $56 \triangle, \square$; from ref $85 \Delta, \boldsymbol{\square}$; from ref $83 \bigcirc, \bigcirc$; from ref $33 \nabla$

should equal $(1-\lambda)_{d}$. This expectation is actually fulfilled as is illustrated by the data of Figure 6 for a variety of different type polyethylene samples. Here, the results for both regular and irregular structured chains are presented. The level of crystallinity for these samples range from 0.25 to greater than 0.90 . The pure orthorhombic crystallinity, as represented by $\alpha_{c}$, plus the interfacial content is equal to the degree of crystallinity as measured by the density. It is possible to obtain a wide range in the interfacial content by control of molecular weight and chain structure. This quantity is apparently not greatly influenced by the crystallization conditions.

\section{Crystallite Thickness}

The crystallite thickness can be determined from either small-angle X-ray scattering or the analysis of the Raman low frequency longitudinal acoustic mode (LAM) ${ }^{58-60}$ The X-ray method requires that the measured periodicity be corrected for the degree of crystallinity. The lamellae also need to be stacked in a reasonably regular array in order to observe a diffraction maximum. This condition is not always achieved. ${ }^{61}$ When the Raman method is applied properly the crystallite size distribution is directly obtained. ${ }^{62,63}$ The complexities that have been introduced into the basic theory ${ }^{64-66}$ have been shown to be unwarranted for the polyethylenes. ${ }^{60,63}$
They have merely served to overcomplicate a relatively straightforward problem. The applicability of the simple procedures to other polymers is a matter of current study. The LAM yields the distribution of ordered sequence lengths. When corrected for the chain tilt the crystallite or "core" thickness is derived. Using Snyder's analytical method ${ }^{62}$ it has been successfully applied to ethylene homopolymers and copolymers crystallized from either bulk or dilute solution. ${ }^{33,60}$

The crystallite thicknesses are very sensitive to the mode of crystallization ${ }^{19,69,70}$ and to the structural regularity of the chain. For rapid, non-isothermal crystallization of linear polyethylene the results are dependent on the sample thickness. For $200 \mu \mathrm{m}$ films the crystallite thickness is about $135-150 \AA$ and is independent of molecular weight. ${ }^{71}$ This thickness is only very slightly dependent on the crystallization temperature. Electron microscope studies have confirmed these conclusions. ${ }^{18,19}$

The larger crytallite thicknesses are obtained after isothermal crystallization. The question as to whether the lamellar thickness increases, and the distribution changes, during isothermal crystallization had been a matter of controversy for some time. ${ }^{69}$ However, it has been established by several recent works ${ }^{69-73}$ that substantial changes in the crystallite thickness do in fact take place during isothermal crystallization. One recent study claiming the constancy of crystallite thickness is apparently in error. ${ }^{74}$ The mechanism of crystallite thickening is complex and will be discussed in detail elsewhere. ${ }^{75}$ The lamellar thickness changes continuously during crystallization. Therefore, a very broad size distribution usually results. This fact is demonstrated by the Raman LAM analysis $^{69,70}$ and by electron microscopic studies. ${ }^{18,19}$ The rate of thickening, as well as the magnitude is very dependent on the molecular weight and crystallization temperature. We find, therefore, that in the linear polyethylenes crystallite thicknesses which range from about 100 to more than $1000 \AA$ can be developed. The larger sizes are invariably characterized by a very broad distribution. ${ }^{69,70}$

The introduction of non-crystallizing structural irregularities into the chain restricts the crystallite thickness. For example, the presence of only $0.6 \mathrm{~mol} \%$ of branch groups reduces the largest size observed to about $125 \AA$. Figure 7 is a compilation 


\section{MANDELKERN}

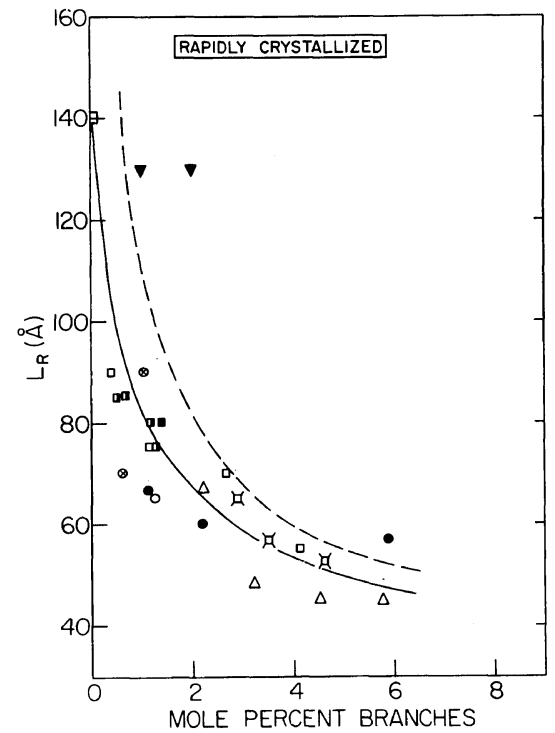

Figure 7. Plot of most probable crystallite thickness, $L_{\mathrm{R}}$, in Angstroms against mole percent branches. Solid line rapidly crystallized samples. From ref 33 hydrogenated polybutadiene $\Delta$, ethylene-butene $\square$, ethylene-octene $\square$; from ref 83 ethylene-vinyl acetate $\mathbf{O}$, branched polyethylene $\bigcirc$; from ref 85 branched polyethylene $\otimes$, ethylene-octene $\square$, ethylene-propylene $\boldsymbol{\nabla}$; from ref 99 ethylene-methacrylic acid . Dashed line represents slowly cooled samples, data not given.

of the results that have been obtained for s:ructurally irregular polyethylene chains. Here the most probable crystallite thickness $L_{\mathrm{R}}$ is plotted against the $\mathrm{mol} \%$ branches. The symbols and solid curve represent rapidly crystallized samples; the dashed line slow cooled samples. ${ }^{* 3}$ Except for these chains with directly bonded methyl groups the crystallite thickness, for a given crystallization mode, depends only on composition and not on the specific chemical nature of the co-units. The initial introduction of co-units into the chain causes a rapid decrease in the crystallite thickness. This size reaches an essentially constant value at about $3.5 \mathrm{~mol} \%$ branching content. It is interesting to note (see below) that in this range of co-unit composition lamellar-like crystallites are no longer observed by electron microscopy.

Since a substantial proportion of the methyl branches enter the crystal lattice on an equilibrium basis the selection of sequences which participate in the crystallization is not as severe as with the other copolymers. This fact is immediately reflected in the observation that the crystallite thickness is comparable to that for the homopolymer under similar crystallization conditions. Other properties, including mechanical ones, ${ }^{77}$ are influenced in a similar manner by the presence of bound methyl groups.

The dashed curve in Figure 7, representing slowly crystallized samples, indicates that only modest changes in thickness take place with changing crystallization conditions. These changes are mainly restricted to the sample with smaller co-unit content. For the higher co-unit containing samples there is essentially no change within the experimental error. In contrast to homopolymers, where the crystallization conditions can cause the crystallite thickness to change by a factor of ten, only very modest differences are observed with copolymers. Major differences in thickness cannot be developed in copolymers by varying either co-unit content or the crystallization mode.

In summary, for the polyethylenes, depending on the chain structure and crystallization conditions under atmospheric pressure, the crystallite thickness can be varied from about $40-50 \AA$ to several thousand $\AA$. The larger sizes are always found in a broad distribution. Thus, a very wide range in this structural variable can be achieved and controlled.

\section{Character of Lamellar Crystallites}

Although a lamellar crystallite habit is a well established characteristic of homopolymer crystallization significant differences are found in their detailed structure with changes in molecular weight and crystallization conditions. ${ }^{17-19,52,78}$ Quantitative electron microscopic studies have revealed that when linear polyethylene is isothermally crystallized at low undercoolings large, geometrically well developed crystallites form with lower molecular weight samples. ${ }^{17-19}$ With increasing molecular weight the lamellae become more curved, are segmented internally and their lateral extent is reduced. For the highest molecular weight studied

*3 During the isothermal crystallization of copolymers only relatively small amounts of crystallinity develop over many decades of time at the crystallization temperature. Since a significant amount of crystallinity is formed on cooling, the interpretation of the structural variables characteristic of such systems is complex. ${ }^{76}$ Hence this mode of crystallization which has been so important to the study of homopolymers, is not discussed here. 
in detail, of the order of $10^{6}$, only small lamellae are observed. When the crystallization temperature is lowered the lamellae become more curved and their lateral extent is severely reduced. The angle of inclination between the chain axis and the normal to the basal plane (the tilt angle) increases with decreasing crystallization temperature. Since the details of chain reentry to the lamellae are dependent on the tilt angle ${ }^{48,49}$ the interfacial free energy associated with the basal plane should be altered. The implication to properties of these kinds of structural changes, while the lamellar habit is maintained, still remains to be investigated.

Surprisingly, well defined lamellae are also observed for compositional fractions of random copolymers containing as much as $3.2 \mathrm{~mol} \%$ of branch points. ${ }^{35,79}$ Although the lamellae for the lower co-unit content copolymers are quite flat their lateral extent is restricted when compared with homopolymer crystallites. The lamellae of copolymers with higher co-unit content are very curve, their lateral extent is restricted and they have become severely segmented. However, the lamellar character can still be identified. For the copolymers which contain a larger concentration of structural irregularities, although crystalline regions can still be detected by electron microscopic techniques, lamellae are no longer formed. Thus starting with the homopolymer, as the co-unit content is increased a continuous degradation in the lamellar character takes place.

\section{Supermolecular Structure}

The discussion of supermolecular structure is concerned with the assembly of the lamellar-like crystallites into higher levels of organization. For example, spherulites represent a particular type of supermolecular structure. The formation of spherulites has been thought to be the universal mode of homopolymer crystallization. Recent studies have shown, however, that the specific supermolecular structure that forms depends in a very definite manner on the molecular weight, crystallization conditions and structural regularity of the molecule. ${ }^{3,76,79,80}$ Small angle light scattering ${ }^{10,80}$ complemented by direct microscopic observation, is a very convenient and objective way to characterize the superstructures. It has been found that the polyethylenes display five different characteristic light scattering patterns. ${ }^{10,80}$ These can be related by theory to corresponding supermolecular forms. ${ }^{81,82}$ The structures observed vary from welldeveloped spherulites, to rod and sheet like aggregates as well as to a random collection of lamellae. The latter clearly do not represent any definite morphological form. Hence spherulitic structures are not always found. It should be recognized that despite their wide spread occurrence and intensive study, spherulites do not represent a universal mode of polymer crystallization.

The supermolecular structures that are observed in linear polyethylene develop in a very systematic manner as the molecular weight and crystallization conditions are varied. The results can be expressed in terms of a morphological map. ${ }^{79,80}$ Under isothermal conditions, in the low molecular weight region, thin rods or "axialites" are formed. At somewhat higher molecular weights, rods whose length is comparable to their breadth, or sheet like structure, develop. At the low temperature boundary for isothermal crystallization, at these molecular weights, spherulites form whose structure become poorer as the molecular weight is increased. For high molecular weights, $M>2 \times 10^{6}$, in the isothermal region the lamellae are always randomly arranged although the level of crystallinity is still of the order of 0.50 to 0.60 . For non-isothermal crystallization the same kind of morphological forms are observed. However, the random type morphology is now found for molecular weights as low as $1 \times 10^{5}$ after crystallization at very low temperatures. Well-developed spherulites are generated in low molecular weight fractions under these same crystallization conditions. Studies with molecular weight fractions of polyethylene oxide show that this polymer displays a very similar morphological pattern. ${ }^{3}$

A wide array of superstructures are formed in polymers in a very systematic way. For the same molecular weight a variety of morphological forms are observed, depending on the crystallization condition. ${ }^{76,79,80}$ In favorable circumstances it is also possible to generate different superstructures from the same molecular weight fraction, each of which has the same level of crystallinity. Therefore, in the study of properties, one now has the capability to isolate the influence of supermolecular structure from the other structural variables.

The characteristics of the morphological maps are altered when irregularities are introduced into 


\section{MANDELKern}

the chain. As a general observation, as far as the supermolecular structure is concerned, a copolymer, or branched polymer, behaves as though it were of much higher molecular weight relative to its linear counterpart. For a given copolymer there is a limited temperature range within which spherulites, of differing degrees of order, can be formed. When the superstructure is examined as a function of molecular weight and crystallization temperature a dome-shaped curve, representing the boundary for spherulite formation evolves. ${ }^{78}$ Within the dome spherulites are observed. At higher and lower temperatures, beyond the dome boundary, random lamellae are usually found. Increasing either the molecular weight or co-unit content reduces the tendency to spherulite formation and favors a random arrangement of the lamellae.

In the preceeding discussion we have assessed the influence of chain structure and crystallization conditions on a set of key independent variables which quantitatively describe the semicrystalline state. These variables can be carefully controlled and can assume a wide range of values. Therefore, experiments can be designed which isolate these variables and allows them to be related to specific properties. Following this approach we next analyze a variety of properties in terms of these structural variables.

\section{PROPERTIES}

A large number of properties have been found to depend primarily on the level of crystallinity. These include the density, enthalpy of fusion, infrared absorption, Raman internal modes, the intensity of both wide and small angle X-ray scattering, the intensity of certain dynamic mechanical relaxations, proton broadline NMR, carbon-13 NMR, and the yield stress to cite but a few and diverse examples. ${ }^{9}$ In some specific cases there are also direct contributions from the interface and the molecular weight. The level of crystallinity thus represents a very important independent structural variable as far as properties are concerned.

The extensive studies that have been made of spherulites, leads naturally to the expectation that the supermolecular structure should be a very important variable in determining properties. This problem can now be addressed since it is possible to isolate the contributions from the superstructure. Studies have shown that these kind of structures have very little influence on thermodynamic ${ }^{80}$ and spectral properties ${ }^{56}$ as well as the mechanical properties that have been studied from this point of view. ${ }^{83,84}$ The important factors appear to be the details of the lamellar structure and the associated interfacial region as well as the conformation of the chain units which connect crystallites. The organization of the lamellae does not appear to play a very crucial role. The nature and the size of the superstructure must of course affect optical properties, particularly in thin films.

Some complex phenomena can be analyzed by the approach that has been adopted. One example is the carbon-13 crystalline spin lattice relaxation time, $T_{1}$. The very large variation in the reported values for $T_{1}$ of the polyethylenes has engendered a great deal of confusion. A recent study has shown that $T_{1}$ in the polyethylenes can vary from about $40 \mathrm{sec}$. to $4500 \mathrm{sec}^{85}$ These admittedly represent a very large range in the values of a relaxation time. The reason for this unusual result is that the $T_{1}$ 's are shown to be a monotonic function of the crystallite thickness when the interface is characteristic of bulk crystallized polymers. However, if this interfacial structure is removed then $T_{1}$ increases five to six fold. The other independent structural variables do not affect $T_{1}{ }^{85}$

Several of the dynamic mechanical relaxations, characteristic of the polyethylenes, have also been studied. ${ }^{83,86}$ The polyethylenes as a class display a set of such transitions. In the order of decreasing temperature, below the melting temperature, the main ones have been designated as the $\alpha$ and $\beta$ transitions or relaxations. Although these transitions have been extensively studied there has been a great deal of uncertainty as to their structural and molecular origin. This difficulty is undoubtedly associated with complications introduced by crystallinity.

The $\alpha$ and $\beta$ transitions have been analyzed in terms of the structural variables important to the crystalline state. ${ }^{83,86}$ The $\alpha$-transition is a well known relaxation assigned to the crystalline regions of polyethylene and can be observed by either dynamic mechanical or dielectric measurements. ${ }^{87}$ The transition temperature varies from $30^{\circ}$ to $120^{\circ} \mathrm{C}$, depending on the polymer type and mode of crystallization. It had been suggested that the transition temperature is governed by the crystallite thickness. ${ }^{88}$ Direct measurement of the crystallite 
thickness, by the Raman LAM techniques, has confirmed this postulate. ${ }^{83}$ The range in observed transition temperatures thus receives a natural explanation. The $\alpha$-transition temperature is independent of the level of crystallinity and the supermolecular structure. ${ }^{83}$ Consideration of these results together with the factors which influence the crystalline carbon-13 $T_{1}$ 's, strongly suggests that there is a coupling of the motion within the crystallite with that of the interface. ${ }^{83,89}$

In addition to the main transition, a minor or second transition, designated $\alpha_{1}$, is occasionally reported. There apparently is no consistent pattern for the observation of this transition. It is usually found as either a shoulder, or a weak peak, in the relaxation spectrum. Since the main $\alpha$-transition temperature is controlled by the crystallite thickness it is reasonable to propose that extremes in this quantity should give rise to other transitions of this type. The results of specifically designed experiments, wherein the size distribution was varied, support this hypothesis. ${ }^{83}$ There is, therefore, a very natural and simple explanation for the existence of the $\alpha_{1}$ transition as well as the fact that it is not always observed. These conclusions do not preclude the possibility that there may be other mechanisms, leading to other transitions, in this temperature region..$^{90,91}$

The $\beta$-transition, which is usually found in the range of $-30^{\circ} \mathrm{C}$ to $+10^{\circ} \mathrm{C}$ is another important relaxation found in the polyethylenes. ${ }^{87}$ Although this transition requires the presence of crystallinity it is not characteristic of motion within the crystalline region. This transition is very intense for polyethylene chains which contain structural irregularities. ${ }^{87}$ However, its existence in the linear polyethylenes has been in dispute, with contrary evidence being reported in the literature. ${ }^{83}$ The intensity of the transition for the branched polymer and for copolymers has lead to its being identified with the glass temperature of all the polyethylenes. ${ }^{92}$ However, its invariance with composition for a given co-unit type (see below), as well as the correlation time for segmental relaxation that is deduced from carbon-13 NMR measuremeirts ${ }^{93,93 a, 93 b}$, rule out this interpretation.

Experimental studies have shown that the $\beta$ transition temperature is independent of crystallite thickness, supermolecular structure and level of crystallinity ${ }^{83}$ However, it has been demonstrated that an interfacial content of at least $10-15 \%$ is necessary for this transition to be observed. ${ }^{87}$ This finding explains the apparent elusiveness of this transition in linear polyethylene. By referring to Table I we recall that for the lower molecular weights the interfacial content is relatively small. It becomes $10 \%$ or greater at the higher molecular weights. Careful examination of the literature reports show that these facts are the reason for the divergence of results. Those investigators who studied lower molecular weight samples reported that no transition existed. On the other hand, those who reported a transition always studied high molecular weight samples. Thus, there is a self-consistency to the observations with linear polyethylene. The location of the $\beta$-transition temperature for linear polyethylene has been established at about $-20^{\circ} \mathrm{C}$.

Following the arguments set forth above the very intense $\beta$-transition observed with the structurally irregular polyethylenes is given a natural explanation. As we have found, such systems always have a relatively high interfacial content. Thus, based upon the molecular interpretation, a well defined $\beta$ transition is to be expected. The basis for the $\beta$ transition in the polyethylenes should also apply to other semi-crystalline polymers and copolymers. This expectation has been fulfilled in copolymers of polyethylene oxide. ${ }^{94,95}$

Ethylene copolymers follow a well-known and complex transition temperature-composition relation. In brief summary, at high co-unit content the completely amorphous copolymers display a conventional glass temperature which follows the usual composition relation. However, at co-unit contents where crystallinity develops the $\beta$-transition is observed. Furthermore, this transition temperature is invariant with composition. Its location depends on the specific co-unit type which in turn determines the temperature at which crystallinity first develops. It can, therefore, be positioned above or below the $\beta$-transition of the linear polymer, as is in fact observed. ${ }^{86}$ The invariance is due to the fact that the relaxation is governed by the interfacial structure. At the extreme of high ethylene content the $\beta$ transition has to approach that of linear polyethylene. Hence, in this composition region it will, in general, vary with composition.

A similar strategy has been used to investigate the stress-strain behavior and the ultimate properties of 


\section{MANDELKeRN}

crystalline polymers. ${ }^{84}$ The initial modulus, the yield stress, the draw ratio (strain) and tensile stress at break of the polyethylenes have been examined in this manner. For the linear polymer the draw ratio at ambient temperature is a monotomically decreasing function of molecular weight and can be varied by a factor of about 10 . Neither the level of crystallinity, for a given molecular weight, nor the supermolecular structure influence the ultimate draw ratio. ${ }^{84}$ The major controlling factor is the molecular weight. As has been pointed out the molecular weight determines the structure, or topology, of the residual non-crystalline regions. By referring back to Figure 1 we find a close connection between the structural factors which govern this mechanical property and those which determine the level of crystallinity. Both are controlled by the residual structure of the melt and depend strongly on molecular weight. Flory and Yoon ${ }^{96}$ have pointed out that the structure of this region is very important to deformation processes. From these results it is deduced that the "unfolding" of chains, or the disruption of mosaic blocks, ${ }^{97}$ which have been postulated to play a key role are not very important. The tensile stress at break depends on molecular weight in a very similar manner.

Studies which encompass the complete range in crystallinities that can be attained with the polyethylenes show that the yield stress depends primarily on the crystallinity level. ${ }^{84}$ There is some indication that at the very high molecular weights there is also a direct influence of chain length. The initial Young's modulus, which plays an important role in the deformation can be varied more than tenfold. It does not depend in any simple manner on the degree of crystallinity, and bears no relation to the supermolecular structure. It depends on the molecular weight and crystallite thickness. As these few examples illustrate, by following these procedures it is becoming possible to define the structural factors which determine a given mechanical property.

In summary we can conclude that although semicrystalline polymers represent a complex structural situation it is possible to sort out the key independent variables which influence important properties. These structural considerations pervade all phenomena and properties involving semi-crystalline polymers, including many not discussed here. Following this strategy gives us the capacity to handle problems of greater and greater complexity.

Acknowledgment. This work was supported in part by the National Science Foundation, Polymers Program, Grant DMR-83-14679 to Florida State University. The assistance of Drs. R. Alamo and K. Anandakumar in preparing the compilations is gratefully acknowledged.

\section{REFERENCES}

1. E. Ergöz, Ph. D. dissertation, Florida State University, December 1970.

2. E. Ergöz, J. G. Fatou, and L. Mandelkern, Macromolecules, 5, 147 (1972).

3. R. C. Allen and L. Mandelkern, J. Polym. Sci., Polym. Phys. Ed., 20, 1465 (1982).

4. P. Geil, "Polymer Single Crystals," WileyInterscience, New York, N. Y., 1963.

5. H. A. Stuart, Ann. N. Y. Acad. Sci., 83, 3 (1959).

6. P. H. Lindenmeyer, Science, 147, 1256 (1956).

7. J. D. Hoffman, Soc. Plastics Eng., Trans., 4, 315 (1964).

8. L. Mandelkern, J. Phys. Chem., 75, 3290 (1971).

9. L. Mandelkern, "Proceedings of the 20th Army Materials Research Conference," Syracuse University Press, 1975.

10. L. Mandelkern, Faraday Soc., Discuss., 68, 310 (1979).

11. J. Schelten, D. H. G. Ballard, G. D. Wignall, G. Longman, and W. Schwartz, Polymer, 17, 751 (1976).

12. D. Y. Yoon and P. J. Flory, Polymer, 18, 509 (1977).

13. D. Y. Yoon and P. J. Flory, Faraday Soc. Discuss., 68, 288 (1979).

14. M. Stamm, E. W. Fischer, M. Dettenmaier, and P. Convert, Faraday Soc. Discuss., 68, 263 (1979).

15. G. Kanig, Prog. Colloid Polym. Sci., 57, 176 (1975).

16. G. R. Strobl, H. Schneider, and I. G. Voigt-Martin, J. Polym. Sci., Polym. Phys. Ed., 18, 1361 (1980).

17. I. G. Voigt-Martin, E. W. Fischer, and L. Mandelkern, J. Polym. Sci., Polym. Phys. Ed., 18, 2347 (1980).

18. I. G. Voigt-Martin and L. Mandelkern, J. Polym. Sci., Polym. Phys. Ed., 19, 1769 (1981).

19. I. G. Voigt-Martin and L. Mandelkern, J. Polym. Sci., Polym. Phys. Ed., in press.

20. R. C. Allen and L. Mandelkern, to be published.

21. E. Lovering, J. Polym. Sci., C, 30, 329 (1970).

22. J. H. Magill, private communication.

23. J. G. Fatou and L. Mandelkern, J. Phys. Chem., 69, 417 (1965).

24. N. Bekkedahl and H. Matheson, J. Res. Natl. Bur. Stand., 15, 503 (1935).

25. L. Mandelkern, "Crystallization of Polymers," McGraw-Hill, New York, N. Y., 1964, p 306. 
26. P. Calvert, J. Polym. Sci., Polym. Phys. Ed., 17, 1341 (1979).

27. M. J. Richardson, P. J. Flory, and J. B. Jackson, Polymer, 4, 221 (1963).

28. P. J. Flory, J. Chem. Phys., 17, 223 (1949)

29. P. J. Flory, Trans. Faraday Soc., 51, 848 (1955).

30. C. H. Baker and L. Mandelkern, Polymer, 7, 7 (1966).

31. G. R. Strobl and W. Hagedorn, J. Polym. Sci., Polym. Phys. Ed., 16, 1181 (1978).

32. M. Glotin and L. Mandelkern, Colloid Polym. Sci., 260, 182 (1982).

33. R. Alamo, R. Domszy, and L. Mandelkern, submitted for publication.

34. L. Mandelkern, A. L. Allou, Jr., and M. Gopalan, J. Phys. Chem., 72, 309 (1968).

35. I. G. Voigt-Martin and L. Mandelkern, to be published.

36. A. Keller, Makromol. Chem., 34, 1 (1959), Polymer, 3, 393 (1962).

37. J. D. Hoffman and J. K. Lauritzen, J. Res. Natl. Bur. Stand. Sect. A, 64, 73 (1960); ibid., 65, 297 (1961).

38. F. C. Frank and M. Tosi, Proc. R. Soc. London, Ser. A, 263, 323 (1961).

39. F. P. Price, J. Chem. Phys., 35, 1884 (1964).

40. F. P. Price, "Nucleation," A. C. Zettlemoyer, Ed., Marcel Dekker, New York, N. Y., 1969, p 405.

41. P. D. Calvert and D. R. Uhlmann, J. Appl. Phys., 43, 944 (1972).

42. H. G. Zachmann, Pure Appl. Chem., 38, 79 (1974).

43. L. Mandelkern, "Crystallization of Polymers," McGraw Hill, New York, N. Y., 1964, p $215 f f$.

44. M. Tasumi and S. Krimm, J. Polym. Sci., A-2, 6, 995 (1968); M. Burk and S. Krimm, ibid., 7, 1785 (1969).

45. X. Jing and S. Krimm, J. Polym. Sci., Polym. Lett. Ed., 21, 123 (1983).

46. G. D. Wignall, L. Mandelkern, C. Edwards, and M. Glotin, J. Polym. Sci., Polym. Phys. Ed., 20, 245 (1982)

47. P. J. Flory, J. Am. Chem. Soc., 84, 2857 (1962).

48. P. J. Flory, D. Y. Yoon, and K. A. Dill, Macromolecules, 17, 862 (1984).

49. D. Y. Yoon and P. J. Flory, Macromolecules, 17, 868 (1984).

50. L. Mandelkern, J. M. Price, M. Gopalan, and J. G. Fatou, J. Polym. Sci., A-2, 4, 385 (1966).

51. J. M. Schultz, W. H. Robinson, and G. M. Pound, J. Polym. Sci., A-2, 5, 511 (1967).

52. G. M. Stack, L. Mandelkern, and I. G. VoigtMartin, Macromolecules, 17, 34 (1984).

53. K. Bergmann and K. Nawotki, Kolloid Z.-Z. Polym., 219, 132 (1967).

54. R. Kitamaru, F. Horii, and S. H. Hyon, J. Polym. Sci., Polym. Phys. Ed., 15, 821 (1977).

55. G. R. Strobl and W. Hagedorn, J. Polym. Sci., Polym. Phys. Ed., 16, 1181 (1978).

56. M. Glotin and L. Mandelkern, Colloid Polym. Sci.,
260, 182 (1982).

57. M. Glotin, R. Domszy, and L. Mandelkern, J. Polym. Sci., Polym. Phys. Ed., 21, 285 (1983).

58. S. Mizushima and T. Shimanouchi, J. Am. Chem. Soc., 71, 1320 (1949).

59. G. R. Strobl and R. Eckel, J. Polym. Sci., Polym. Phys. Ed., 14, 913 (1976).

60. M. Glotin and L. Mandelkern, J. Polym. Sci., Polym. Phys. Ed., 21, 29 (1983).

61. I. G. Voigt-Martin, E. W. Fischer, W. Hagedorn, P. Hendra, L. Mandelkern, and K. Mehler, The Proceedings the 26th IUPAC Macromolecules, Mainz, 1979, p 1350.

62. R. G. Snyder, S. J. Kruase, and J. R. Scherer, J. Polym. Sci., Polym. Phys. Ed., 16, 1593 (1978); ibid., 18, 421 (1980).

63. M. Glotin and L. Mandelkern, J. Polym. Sci., Polym. Lett. Ed., 21, 807 (1983).

64. S. L. Hsu and S. Krimm, J. Appl. Phys., 48, 4013 (1977).

65. C. Chang and S. Krimm, J. Polym. Sci., Polym. Phys. Ed., 17, 2163 (1979).

66. A. Peterlin, J. Appl. Phys., 50, 838 (1979); J. Mater. Sci., 14, 2994 (1979).

67. R. C. Domszy, R. Alamo, P. J. M. Mathieu, and L. Mandelkern, J. Polym. Sci., Polym. Phys. Ed., in press (1984).

68. R. C. Domszy, M. Glotin, and L. Mandelkern, $J$. Polym. Sci., Polym. Symp. Ed., in press (1984).

69. G. M. Stack, L. Mandelkern, and I. G. VoigtMartin, Polym. Bull., 8, 421 (1982).

70. G. M. Stack, Ph. D. dissertation, Florida State University, August 1983.

71. P. J. M. Mathieu and L. Mandelkern, to be published.

72. J. Dlugosz, C. Fraser, D. Grubb, A. Keller, J. Odell, and P. Goggin, Polymer, 17, 471 (1976).

73. P. J. Barham, R. A. Chivers, D. A. Jarvis, J. Martinez-Salajar, and A. Keller, J. Polym. Sci., Polym. Lett. Ed., 19, 539 (1981); ibid., 20, 1717 (1982).

74. W. D. Varnell, I. R. Harrison, and J. I. Wang, J. Polym. Sci., Polym. Phys. Ed., 19, 1577 (1981).

75. L. Mandelkern and G. M. Stack, to be published.

76. M. Glotin and L. Mandelkern, Macromolecules, 14, 1394 (1981).

77. G. Capaccio and I. M. Ward, J. Polym. Sci., Polym. Phys. Ed., 22, 475 (1984).

78. D. C. Bassett, A. M. Hodge, and R. H. Olley, Faraday Soc. Discuss., 68, 218 (1979).

79. L. Mandelkern, M. Glotin, and R. A. Benson, Macromolecules, 14, 22 (1981).

80. J. Maxfield and L. Mandelkern, Macromolecules, 10, 1141 (1977); ibid., J. Polym. Sci., Polym. Phys. Ed., 17, 1913 (1979).

81. R. S. Stein, "New Methods of Polymer Characterization," B. Ke, Ed., Wiley-Interscience, New 


\section{MANDELKERN}

York, N. Y., 1964.

82. M. Moritani, N. Hayashi, A. Utsuo, and H. Kawai, Polym. J., 2, 74 (1971).

83. R. Popli, M. Glotin, L. Mandelkern, and R. S. Benson, J. Polym. Sci., Polym. Phys. Ed., 22, 407 (1984).

84. R. Popli and L. Mandelkern, to be published.

85. D. E. Axelson, L. Mandelkern, R. Popli, and P. Mathieu, J. Polym. Sci., Polym. Phys. Ed., 21, 2319 (1983).

86. R. Popli and L. Mandelkern, Polym. Bull., 9, 260 (1983).

87. N. G. McCrum, B. E. Read, and G. Williams, "Anelastic and Dielectric Effects in Polymer Solids," Wiley, London, 1967.

88. C. R. Ashcraft and R. H. Boyd, J. Polym. Sci., Polym. Phys. Ed., 14, 2153 (1976).

89. N. G. McCrum, "Molecular Basis of Transition and Relaxation," D. J. Meier, Ed., Gordon and Breech, New York, N. Y., 1978.

90. S. Suehiro, T. Yamada, H. Iragaki, T. Kyu, S. Nomura, and H. Kawai, J. Polym. Sci., Polym. Phys. Ed., 17, 763 (1979).
91. R. J. Cembrola, T. Kyu, S. Suehira, H. Kawai, and R. S. Stein, J. Polym. Phys., Polym. Phys. Ed., 20, 1279 (1982).

92. G. T. Davies and R. K. Eby, J. Appl. Phys., 44, 4274 (1973).

93. D. E. Axelson and L. Mandelkern, J. Polym. Sci., Polym. Phys. Ed., 16, 113 S (1978).

93. a. J. J. Dechter, D. E. Axelson, A. Dekmezian, M. Glotin, and L. Mandelkern, J. Polym. Sci., Polym. Phys. Ed., 20, 641 (1982).

b. A. Dekmezian, D. E. Axelson, J. J. Dechter, B. Borah, and L. Mandelkern, J. Polym. Sci., Polym. Phys. Ed., submitted.

94. L. Bohn, Kolloid-Z., 201, 20 (1965).

95. Y. S. Papir and E. Baer, Mater. Sci. Eng., 8, 310 (1971).

96. P. J. Flory and D. Y. Yoon, Nature, 272, 226 (1978).

97. A. Peterlin, J. Polym. Sci., C, 18, 123 (1967).

98. F. Sakaguchi and L. Mandelkern, Analytical Calorimetry, 4, 95 (1977).

99. K. Anandakumar and L. Mandelkern, to be published. 